\title{
Quelles perspectives pour la lutte génétique vis-à-vis du mildiou du tournesol ?
}

\author{
Denis TOURVIEILLE de LABROUHE \\ Emmanuelle MESTRIES ${ }^{2}$ \\ Pascal WALSER ${ }^{1}$ \\ ${ }^{1} U M R$ « ASP », INRA, \\ 234, av. du Brézet, \\ 63100 Clermont-Ferrand France \\ $<$ tourvie@clermont.inra.fr> \\ ${ }^{2}$ CETIOM, Centre de Grignon, \\ BP 4, \\ 78850 Thiverval-Grignon, \\ France
}

\begin{abstract}
Downy mildew of sunflower exists as physiological races which have changed in France following introductions or selection pressure applied by large-scale use of varieties with single major genes giving resistance to the disease. The duration of efficiency of single or pyramided resistance genes, their alternation over different years or use of mixtures of several isogenic forms of a hybrid with different resistance genes, were studied under six irrigated $60 \mathrm{~m}^{2}$ net cages which made it possible to obtain disease attack whatever the weather conditions. Studies have been made over 4 years of continuous cropping. The susceptible form of the hybrid showed from 37 to $75 \%$ attack, always by race 710 , present at the beginning of the study. Monoculture of the two forms of the hybrid each carrying one efficient resistance gene showed no significant attack in years 1 and 2, but then 4-5\% attack in year 3 and $15-21 \%$ attack in year 4 , with the appearance of races new to the trial plots but which have appeared elsewhere in France where varieties with similar resistances are grown. In contrast, there were no significant attacks on the form of the hybrid carrying both efficient resistance genes. In the cage in which different forms of the hybrid were alternated, after $75 \%$ attack on the susceptible form, cropping of single resistance genes and then of the pyramided form, always gave less than $2 \%$ attack. The cage in which a mixture (25\% of each hybrid form) was grown always showed one quarter of the attack in the cage containing the susceptible hybrid, and very little change in virulence. Thus, pyramiding, alternation or mixtures of a few major genes all appear to increase the duration of effective control of downy mildew. Their possible use on a large scale, the difficulties that would be encountered by both breeders and farmers and combination with other possible methods to limit downy mildew attacks are discussed.
\end{abstract}

Key words: downy mildew, sunflower, plasmopara halstedii, durability, alternation, pyramiding, mixture

blèmes « Mildiou » sur le terrain, dus à l'apparition des races $710(=$ race $A)$ et 703 (= race $B)$ [5]. Depuis cette date, le parasite est sous surveillance et les prospections permettent de déceler son évolution avant l'apparition de dégâts trop importants [6-10]. Les analyses réalisées sur I'ADN des races 703 et 710 ont montré qu'elles étaient plus proches des souches américaines qui présentaient déjà de nombreuses races différentes [11] que des souches françaises qui présentent une forte stabilité de virulence [12]. L'origine de ces deux nouvelles races est donc attribuée à une importation accidentelle de semences contaminées. À partir de ces introductions, les prospections annuelles mettent en évidence l'existence de 3 races en 1993, 5 en 1994, 6 en 2000, 9 en 2002 et finalement pas moins de 12 races en 2004 [13]. Les nouvelles races qui émergent ces quatre dernières années $n$ 'ont pas été décrites dans d'autres pays, elles semblent donc issues de l'évolution des populations installées sur le territoire depuis 1990.

Nous avons [14] expliqué cette évolution en comparant les résultats des prospections qui ont permis aux SPV (services de la protection des végétaux) de suivre l'évolution des virulences, avec les profils de résistance des variétés commercialisées (figure 1). Durant les années 80 , tous les hybrides cultivés en France possédaient le gène de résistance $P / 1$ ou $P / 2$, tous deux efficaces vis-à-vis de la race 100 [15]. Avec I'introduction des races 710 et 703 , la lutte génétique s'est essentiellement axée sur l'utilisation d'un autre groupe de gènes de résistance facile d'accès : Pl6 ou PI7 [16]. Le travail des sélectionneurs a permis d'obtenir rapidement des génotypes résistants aux principales races installées en France. Associé à la souplesse $d^{\prime}$ inscription au catalogue officiel des formes modifiées (variétés identiques à une variété déjà inscrite mais disposant $d^{\prime}$ un gène $P I$ en plus), ce travail considérable fait qu'en 2000 plus de la moitié de la surface emblavée en tournesol l'était avec des variétés possédant un gène du groupe P/6/PI7. C'est à partir de cette date que des races capables de surmonter ces résistances sont apparues.

Dans les conditions de culture françaises (rotations courtes (2-3 ans) et printemps humides), 


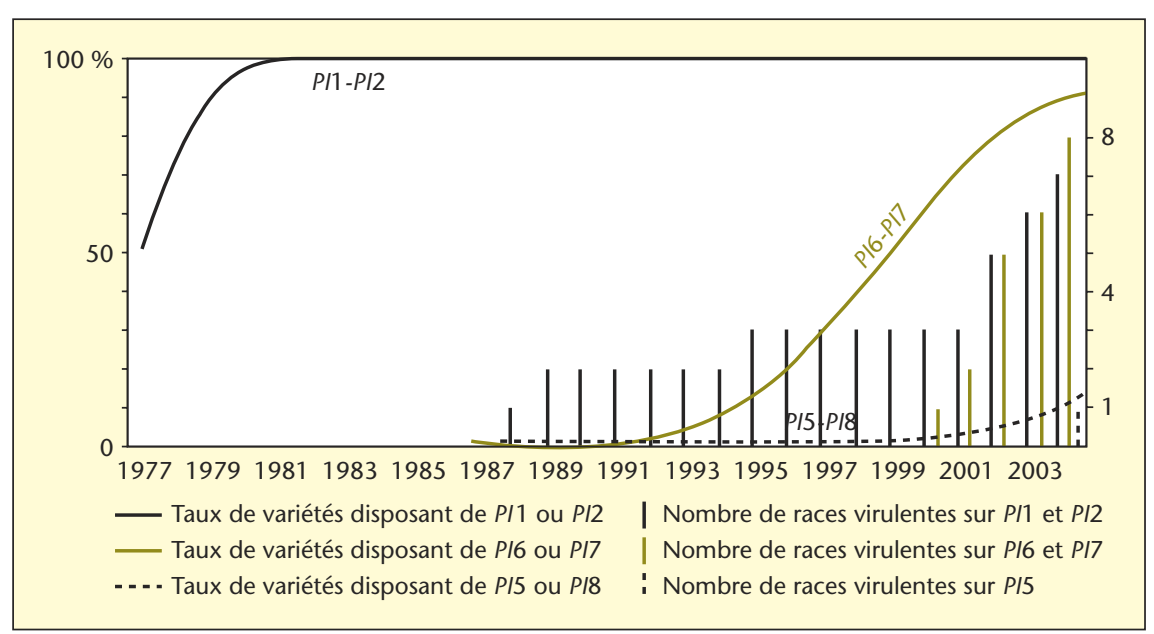

Figure 1. Comparaison entre l'évolution en France du taux d'utilisation des gènes Pl de résistance du tournesol et du nombre de pathotypes de Plasmopara halstedii virulents sur ces gènes entre 1977 et 2004 [13]

I'utilisation quasi systématique d'une seule source de résistance semble conduire rapidement à la « sélection » de nouvelles races qui remettent en cause la lutte génétique. L'utilisation plus récente $d^{\prime}$ autres sources de résistance PI5/P/8 [17] conduit au même constat puisqu'en 2004 des variétés possédant un gène de ce groupe $(P / 5)$ ont subi, pour la première fois en France, des pertes liées à l'apparition d'un nouveau pathotype (race 334) de Plasmopara halstedii [13].

La fréquence de détection des nouvelles races est liée à l'intensité de la culture du tournesol et se situe donc essentiellement dans le sud-ouest et l'ouest de la France (SPV, comm. pers.). Ces observations montrent que dans les conditions de culture françaises, la « durée de vie » d'un gène $P /$ est inférieure à 10 ans.

La course entre l'obtenteur et le parasite tournera probablement à l'avantage de ce dernier par épuisement des gènes facilement utilisables en sélection si de nouvelles stratégies ne sont pas envisagées pour ralentir l'évolution du parasite ou pour en réduire les conséquences économiques. Ces stratégies ont été expérimentées pour freiner les épidémies polycycliques dues à divers parasites aériens. Ainsi, de Vallavieille-Pope [18] montre l'intérêt du mélange de cultivars de céréales qui réduit les niveaux de la maladie du fait de la perte d'inoculum due à la présence des plantes résistantes entre les sensibles. En outre, la résistance induite par les races avirulentes peut réduire I'infection par les races virulentes. McDonald et Linde [19] montrent que, lorsqu'un gène majeur de résistance est réparti sur une grande zone géographique, cela entraîne une augmentation de la fréquence des mutants virulents. Deux possibilités s'offrent alors. La plus commune consiste à combiner plusieurs gènes majeurs dans un même cultivar (" pyramidage ») dans l'espoir que l'agent pathogène ne pourra pas présenter simultanément les caractères conduisant à la virulence vis-à-vis de tous les gènes associés. Une autre option consiste à alterner (dans le temps et/ou dans l'espace) I'utilisation des gènes majeurs afin de provoquer des ruptures dans les pressions de sélection favorisant tel ou tel allèle de virulence. Cette dernière stratégie semble avoir été expérimentée avec succès par Zhu et al. [20] dans le cas des maladies du riz.

Ces stratégies (assemblage, combinaison de gènes et alternance) sont aujourd'hui envisageables chez le pathosystème mildiou/ tournesol du fait de la découverte de plusieurs gènes $P /$ efficaces vis-à-vis des principales races présentes en France et de leur localisation sur la carte chromosomique [16, 17, 21-25], ce qui a permis le développement d'outils (marqueurs) d'aide à la sélection. Dans cet article, nous présenterons les résultats d'un essai pluriannuel mettant en évidence l'impact de l'assemblage, de l'alternance ou de la combinaison de gènes sur les taux de plantes malades et sur l'évolution d'une population de Plasmopara halstedii dans un dispositif contrôlé proche des conditions de culture agricoles. Les méthodes de gestion des gènes majeurs seront discutées et leurs conséquences sur les partenaires de la filière seront analysées.

\section{Matériel et méthode}

\section{Situation sanitaire}

Les parcelles utilisées pour notre expérimentation située sur le domaine expérimental de I'INRA à Clermont-Ferrand, ont été suivies depuis plusieurs années. Les génotypes cultivés sur ces parcelles ne possédaient pas de gène de résistance au mildiou. Les analyses des isolats récoltés (données non fournies) ont montré que ces parcelles n'étaient infestées que par deux pathotypes : race 100 et race 710 .

\section{Génotypes de tournesol}

Quatre hybrides quasi-isogéniques ont été utilisés. Ils ne se distinguent que par les gènes de résistance au mildiou, ils ont été construits à partir de différentes formes des mêmes lignées possédant diverses sources de résistance :

L1 forme a : possédant une source de résistance apportée par la lignée RHA274 (lignée USDA résistante à la race 100).

$\mathrm{L} 1$ forme $b$ : possédant les sources de résistances apportées par RHA274 et RHA340 (lignées USDA résistantes aux races 100 et 710 ).

L2 forme a: ne possédant aucun gène de résistance connu.

L2 forme $b$ : possédant une source de résistance apportée par HA335 (lignée USDA résistante aux races 100 et 710 ).

Les hybrides ont été construits de la manière suivante :

$-\mathrm{H} 1=\mathrm{L} 1$ forme $\mathrm{a} \times \mathrm{L} 2$ forme $\mathrm{a}$

$-\mathrm{H} 2=\mathrm{L} 1$ forme $\mathrm{a} \times \mathrm{L} 2$ forme $\mathrm{b}$

$-\mathrm{H} 3=\mathrm{L} 1$ forme $\mathrm{b} \times \mathrm{L} 2$ forme $\mathrm{a}$

$-\mathrm{H} 4=\mathrm{L} 1$ forme $\mathrm{b} \times \mathrm{L} 2$ forme $\mathrm{b}$

Seul I'hybride H4 combine deux résistances efficaces vis-à-vis des deux pathotypes présents sur les parcelles d'expérimentation.

La caractérisation des profils de virulence des souches de Plasmopara halstedii a été réalisée à I'aide des 9 lignées hôtes-différentiels officielles D1 à D9 qui permettent la dénomination internationale [2] auxquelles nous avons rajouté 3 lignées qui nous semblaient pertinentes: YVQ (lignée INRA résistante à la race 710 mais sensible à la race 100); XRQ (lignée INRA résistante à toutes les races françaises connues en 2003) et RHA340 (lignée USDA résistante à toutes les races de mildiou connues en 2004). Le génotype YVQ doit nous permettre d'isoler le pathotype 100 si celui-ci est en mélange avec le pathotype 710 .

\section{Dispositif expérimental}

II consiste en l'implantation de 6 parcelles expérimentales de 300 plantes environ sous tunnel en filet de $9 \times 7 \mathrm{~m}$. Les hybrides sont semés dans des conditions normales de densité (50 000 pieds/ha). L'irrigation est conduite afin de favoriser les infections racinaires puis aériennes : 7 jours après le semis, $100 \mathrm{~mm}$ d'eau sont apportés pour faciliter la réussite des infections au niveau des radicules, puis un arrosage réglé sur un humectostat [26] permet d'assurer des conditions favorables aux contaminations secondaires (présence d'eau libre sur les feuilles). Les plantes sont suivies jusqu'au début de floraison. À ce stade, toutes les plantes saines sont enlevées alors que les plantes malades sont coupées et laissées sur place afin de conserver l'inoculum et que celui-ci suive son 
Tableau 1. Dispositif pluriannuel d'étude de l'impact du mode de gestion des gènes Pl de résistance du tournesol sur l'évolution du mildiou ; H1, H2, H3 et H4 sont 4 hybrides quasi-isogéniques se distinguant par leur construction de résistance à Plasmopara halstedii.

\begin{tabular}{|c|c|c|c|c|c|c|}
\hline \multirow[b]{2}{*}{ Années } & \multicolumn{6}{|c|}{ Parcelles } \\
\hline & Assemblage & Alternance & Référence & Monoculture H2 & Monoculture H3 & Combinaison \\
\hline $\mathrm{n}$ & $\mathrm{H} 1, \mathrm{H} 2, \mathrm{H} 3, \mathrm{H} 4$ & $\mathrm{H} 1$ & $\mathrm{H} 1$ & $\mathrm{H} 2$ & $\mathrm{H} 3$ & $\mathrm{H} 4$ \\
\hline$n+1$ & $\mathrm{H} 1, \mathrm{H} 2, \mathrm{H} 3, \mathrm{H} 4$ & $\mathrm{H} 2$ & $\mathrm{H} 1$ & $\mathrm{H} 2$ & $\mathrm{H} 3$ & $\mathrm{H} 4$ \\
\hline$n+2$ & $\mathrm{H} 1, \mathrm{H} 2, \mathrm{H} 3, \mathrm{H} 4$ & $\mathrm{H} 3$ & $\mathrm{H} 1$ & $\mathrm{H} 2$ & $\mathrm{H} 3$ & $\mathrm{H} 4$ \\
\hline$n+3$ & $\mathrm{H} 1, \mathrm{H} 2, \mathrm{H} 3, \mathrm{H} 4$ & $\mathrm{H} 4$ & $\mathrm{H} 1$ & $\mathrm{H} 2$ & $\mathrm{H} 3$ & $\mathrm{H} 4$ \\
\hline
\end{tabular}

cycle naturel d'évolution. Chaque parcelle expérimentale est alors immédiatement travaillée individuellement et séparément dans le temps afin d'enterrer l'inoculum et de préparer le terrain pour le semis de l'année suivante. Le dispositif pluriannuel est présenté dans le tableau 1, l'assemblage est constitué d'un mélange à parts égales des quatre hybrides.

\section{Caractérisation des virulences et test de résistance}

Les prélèvements sont réalisés à un stade suffisamment avancé pour ne pas détruire entièrement la plante : à partir du stade 4 paires de feuilles (4PF) jusqu'au stade bouton étoilé (BE). Par plante contaminée, 1 à 2 feuilles présentant une sporulation importante à la face inférieure sont prélevées, le parasite est multiplié sur une variété population ne disposant d'aucun gène de résistance au mildiou, puis la caractérisation des virulences est réalisée sur la série de lignées différentielles en respectant le protocole décrit par Tourvieille de Labrouhe et al. [27]. Nous avons utilisé le même protocole pour caractériser le comportement des quatre hybrides $(\mathrm{H} 1$ à H4) vis-à-vis de la population parasitaire indigène et des souches récoltés durant notre expérimentation. Tous les tests biologiques sont réalisés en enceinte de confinement ayant l'agrément obligatoire pour la manipulation du Plasmopara halstedii (agrément N²003/ DRAF/70).

\section{Résultats}

\section{Caractérisation de la population parasitaire avant expérimentation}

L'analyse de souches monosporanges issues des populations indigènes a mis en évidence seulement deux profils de virulence : races 100 et 710 . Les comportements des quatre hybrides $\mathrm{H} 1$ à $\mathrm{H} 4$ vis-à-vis de ces deux races sont présentés dans le tableau 2.

Des tests réalisés sur les deux races et portant sur une centaine d'individus nous ont permis de préciser le niveau de pureté variétale des quatre hybrides : $\mathrm{H} 1$ (race $100=99 \%$ résistant ; race $710=100 \%$ sensible), $\mathrm{H} 2$ (race $100=99 \%$ résistant ; race $710=96 \%$ résistant), H3 (race $100=99 \%$ résistant; race $710=99 \%$ résistant) et $\mathrm{H} 4$ (100\% résistant aux deux races).

\section{Évolution des taux de plantes malades}

Les observations régulières, une fois par semaine, nous ont permis de distinguer les plantes malades suite à une infection racinaire de celles qui présentaient des symptômes d'infections aériennes systémiques et non systémiques. Les observations réalisées sur les 6 parcelles sont regroupées dans le tableau 3.

\section{Analyse des virulences}

Les tests ont été réalisés sur des isolats représentatifs de la variabilité observée dans les parcelles. Lorsque, pour un traitement donné, le nombre de plantes malades était inférieur à 20, I'analyse a porté sur tous les individus. Lorsque ce nombre était supérieur à 20 , l'analyse a été réalisée sur une sous-population représentative des différentes formes d'attaque: primaire, secondaire systémique et secondaire non systémique. Dans tous les cas, au moins $10 \%$ des plantes malades ont été prélevés et la virulence des isolats caractérisée. Ainsi, au total sur les quatre années de l'expérimentation, 372 prélèvements ont été réalisés qui ont abouti à 362 caractérisations de virulence. Pour 10 prélèvements, essentiellement sur des infections secondaires non systémiques, nous n'avons pas réussi à multiplier le parasite. La répartition des 362 échantillons caractérisés est présentée dans le tableau 4. Sur les 5 pathotypes isolés dans notre expérimentation, seule la race 710

Tableau 2. Caractérisation des pathotypes de Plasmopara halstedii présents l'année $n$ - 1, sur le site d'expérimentation et réponses des 4 hybrides de tournesol : H1, H2, H3 et H4 à cette population indigène. D1-D9 = lignées hôtes différentielles [2], XRQ, YVQ = lignées INRA (France), RHA340 = lignée USDA (USA).

\begin{tabular}{|c|c|c|c|c|c|c|c|c|c|c|c|c|c|c|c|c|}
\hline Génotypes & D1 & D2 & D3 & D4 & D5 & D6 & D7 & D8 & D9 & XRQ & YVQ & RHA340 & $\mathrm{H} 1$ & $\mathrm{H} 2$ & H3 & $\mathrm{H} 4$ \\
\hline Race 100 & 5 & $\mathrm{R}$ & $\mathrm{R}$ & $\mathrm{R}$ & $\mathrm{R}$ & $\mathrm{R}$ & $\mathrm{R}$ & $\mathrm{R}$ & $\mathrm{R}$ & $\mathrm{R}$ & S & $\mathrm{R}$ & $\mathrm{R}$ & $\mathrm{R}$ & $\mathrm{R}$ & $\mathrm{R}$ \\
\hline Race 710 & S & S & 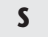 & 5 & $\mathrm{R}$ & $\mathrm{R}$ & $\mathrm{R}$ & $\mathrm{R}$ & $\mathrm{R}$ & $\mathrm{R}$ & $\mathrm{R}$ & $\mathrm{R}$ & $S$ & $\mathrm{R}$ & $\mathrm{R}$ & $\mathrm{R}$ \\
\hline
\end{tabular}

Tableau 3. Evolution du nombre de plantes de tournesol présentant des symptômes dûs à Plasmopara halstedii issus d'infections primaires et secondaires dans chaque parcelle au cours des 4 années d'expérimentation.

\begin{tabular}{|c|c|c|c|c|c|c|c|c|c|c|c|c|c|c|c|c|c|c|c|c|c|c|c|c|}
\hline \multirow{2}{*}{$\begin{array}{l}\text { Parcelles } \\
\text { Années }\end{array}$} & \multicolumn{4}{|c|}{ Référence H1 } & \multicolumn{4}{|c|}{ Assemblage } & \multicolumn{4}{|c|}{ Alternance } & \multicolumn{4}{|c|}{ Monoculture $\mathrm{H} 2$} & \multicolumn{4}{|c|}{ Monoculture $\mathrm{H} 3$} & \multicolumn{4}{|c|}{ Combinaison $\mathrm{H} 4$} \\
\hline & $\mathrm{n}$ & $n+1$ & $n+2$ & $\mathrm{n}+3$ & $\mathrm{n}$ & $n+1$ & $n+2$ & $\mathrm{n}+3$ & $\mathrm{n}$ & $\mathrm{n}+1$ & $\mathrm{n}+2$ & $n+3$ & $\mathrm{n}$ & $\mathrm{n}+1$ & $\mathrm{n}+2$ & $\mathrm{n}+3$ & $\mathrm{n}$ & $\mathrm{n}+1$ & $\mathrm{n}+2$ & $n+3$ & $\mathrm{n}$ & $\mathrm{n}+1$ & $n+2$ & $n+3$ \\
\hline Nbre de plantes total & 284 & 334 & 286 & 322 & 310 & 293 & 332 & 338 & 314 & 355 & 327 & 352 & 377 & 369 & 327 & 351 & 322 & 368 & 322 & 336 & 380 & 365 & 344 & 353 \\
\hline Nbre total de plantes atteintes & 203 & 125 & 215 & 194 & 43 & 19 & 33 & 51 & 236 & 4 & 5 & 4 & 10 & 4 & 16 & 52 & 9 & 4 & 15 & 72 & 0 & 0 & 3 & 3 \\
\hline $\begin{array}{l}\text { Nbre de plantes avec infection } \\
\text { primaire }\end{array}$ & 83 & 46 & 133 & 163 & 38 & 7 & 22 & 46 & 126 & 2 & 2 & 2 & 9 & 2 & 2 & 46 & 8 & 1 & 3 & 40 & 0 & 0 & 0 & 3 \\
\hline $\begin{array}{l}\text { Nbre de plantes avec infection } \\
\text { secondaire systémique }\end{array}$ & 26 & 37 & 72 & 30 & 2 & 10 & 11 & 5 & 27 & 2 & 3 & 2 & 0 & 2 & 14 & 6 & 0 & 0 & 11 & 32 & 0 & 0 & 2 & 0 \\
\hline $\begin{array}{l}\text { Nbre de plantes avec infection } \\
\text { secondaire non-systémique }\end{array}$ & 94 & 42 & 10 & 1 & 3 & 2 & 0 & 0 & 83 & 0 & 0 & 0 & 1 & 0 & 0 & 1 & 1 & 3 & 1 & 0 & 0 & 0 & 1 & 0 \\
\hline
\end{tabular}


Tableau 4. Caractérisation et nombre des pathotypes de Plasmopara halstedii isolés les années $n$ à $n+3$ sur le site d'expérimentation et réponses des 4 hybrides de tournesol : H1, H2, H3 et H4. D1-D9 = lignées hôtes différentielles [2]. XRQ, YVQ = lignées INRA (France). RHA340 = lignée USDA (USA).

\begin{tabular}{|c|c|c|c|c|c|c|c|c|c|c|c|c|c|c|c|c|c|}
\hline Races & Effectifs & D1 & D2 & D3 & D4 & D5 & D6 & D7 & D8 & D9 & XRQ & YVQ & Rha340 & $\mathrm{H} 1$ & $\mathrm{H} 2$ & $\mathrm{H} 3$ & $\mathrm{H} 4$ \\
\hline 304 & 6 & $S$ & $S$ & $\mathrm{R}$ & $\mathrm{R}$ & $\mathrm{R}$ & $\mathrm{R}$ & $\mathrm{R}$ & $\mathrm{R}$ & $S$ & $R$ & $S$ & $\mathrm{R}$ & $\mathrm{R}$ & $\mathrm{R}$ & $\mathrm{R}$ & $\mathrm{R}$ \\
\hline 314 & 20 & S & S & $\mathrm{R}$ & S & $\mathrm{R}$ & $\mathrm{R}$ & $\mathrm{R}$ & $\mathrm{R}$ & S & $\mathrm{R}$ & S & $\mathrm{R}$ & $\mathrm{R}$ & $\mathrm{R}$ & $\mathrm{R}$ & $\mathrm{R}$ \\
\hline 710 & 293 & $S$ & $S$ & S & S & $\mathrm{R}$ & $R$ & $\mathrm{R}$ & $\mathrm{R}$ & $\mathrm{R}$ & $\mathrm{R}$ & $\mathrm{R}$ & $\mathrm{R}$ & S & $R$ & $\mathrm{R}$ & $\mathrm{R}$ \\
\hline 704 & 16 & S & S & S & $R$ & $\mathrm{R}$ & $R$ & $\mathrm{R}$ & $\mathrm{R}$ & S & $R$ & S & $\mathrm{R}$ & S & S & S & S \\
\hline 714 & 27 & $S$ & $S$ & $S$ & $S$ & $\mathrm{R}$ & $R$ & $\mathrm{R}$ & $\mathrm{R}$ & S & $\mathrm{R}$ & S & $\mathrm{R}$ & $S$ & S & S & $S$ \\
\hline
\end{tabular}

avait été identifiée l'année $n-1$ et c'est la seule qui avait été décrite précédemment dans notre région d'expérimentation (SPV, Comm. pers.).

\section{Observations réalisées sur les 6 parcelles}

- Parcelle Référence (monoculture de I'hybride $\mathrm{H} 1)$ : les taux d'attaque observés sont visualisés dans la figure $2 a$. Ils fluctuent du simple: $37,4 \%$ (en année $n+1$ ) au double : 75,2 \% en année $n+2$. De même, les taux d'infection secondaires ne sont pas proportionnels aux taux d'infections primaires, ils varient de $42,3 \%$ la première année à 9,6\% l'année $\mathrm{n}+3$.

Tous les isolats de Plasmopara halstedii, sans exception, appartiennent au pathotype 710 .

- Parcelle Monoculture H2 : les taux d'attaque observés sont visualisés dans la figure $2 b$. Les taux d'attaque, faibles les deux premières années ( $2,7 \%$ et $1,1 \%$ respectivement) augmentent rapidement la troisième année (4,9\%) pour atteindre $15,1 \%$ l'année suivante. Seules 2 plantes présentent des infections primaires l'année $\mathrm{n}+2$.

Tous les individus (14 plantes) infectés les deux premières années l'étaient avec le pathotype 710. La troisième année sur les 16 individus infectés, un tiers (5 plantes) l'est avec la race 714. La dernière année, ce sont deux tiers (7 plantes/11 analysées) qui sont infectés avec la race 714 . Le taux de plantes infectées avec la race 710 est toujours inférieur à 3,5\%.

- Parcelle Monoculture H3 : les taux d'attaque observés sont visualisés dans la figure $2 c$. Le taux d'attaque est faible les deux premières années (2,8 \% et $1,1 \%$ respectivement) puis il augmente rapidement la troisième année (4,6\%) pour atteindre 21,4\% l'année suivante. Seules 3 plantes présentent des infections primaires l'année $\mathrm{n}+2$.

Tous les individus ( 13 plantes) infectés les deux premières années l'étaient avec le pathotype 710. La troisième année, sur les 15 individus infectés, un tiers (5 plantes) l'est avec la race 714 ou 704. Un isolat est caractérisé comme appartenant au pathotype 314. La dernière année, les races 704 ou 714 concernent les deux tiers des analyses (18/31). Le taux de plantes infectées avec la race 710 est toujours inférieur à 3,0 \%.
- Parcelle Combinaison H4 : les taux d'attaque observés sont visualisés dans la figure $2 d$. Les taux d'attaque restent extrêmement faibles, inférieurs à $1,0 \%$.

Les isolats récoltés les années $\mathrm{n}+2$ et $\mathrm{n}+3$ appartiennent aux pathotypes 304 (3 isolats), 704 (2 isolats) et 314 (1 isolat).

- Parcelle Alternance (alternance des hybrides $\mathrm{H} 1, \mathrm{H} 2, \mathrm{H} 3$ et H4) : les taux d'attaque observés sont regroupés dans la figure $2 e$. Le taux d'attaque l'année $n(75,1 \%)$ avec l'hybride H1 est équivalent à celui qui est relevé dans la parcelle de référence $(71,5 \%)$. Les années suivantes, avec successivement les hybrides $\mathrm{H} 2, \mathrm{H} 3$ et $\mathrm{H} 4$, les taux d'attaque restent inférieurs à $2 \%$.

Durant les trois premières années, seule la race 710 a été isolée, en année $n+3$ le pathotype 714 a été isolé à partir d'infections secondaires. - Parcelle Assemblage (monoculture d'un mélange $1 / 4 \mathrm{H} 1,1 / 4 \mathrm{H} 2,1 / 4 \mathrm{H} 3$ et $1 / 4 \mathrm{H} 4$ ) : les taux $d^{\prime}$ attaque observés sont visualisés dans la figure $2 f$. Ils varient du simple au double (de 6,5 à 15,1\%), avec comme pour la parcelle de référence un minimum l'année $n+1$.

Tous les isolats de Plasmopara halstedii analysés, à l'exception de 5 récoltés l'année $n+3$, appartiennent au pathotype 710 (86/91). Parmi ces 5 isolats, deux isolats récoltés sur des plantes infectées au niveau des racines appartiennent au pathotype 304 , alors que les trois autres, récoltés sur des plantes infectées tardivement ont un profil de virulence de type 714 .

\section{Comparaison des taux d'attaque primaire et secondaire dans}

\section{les parcelles Référence et Assemblage}

Les isolats récoltés dans la parcelle de référence (monoculture $\mathrm{H} 1$ ) et dans celle correspondant à l'assemblage (monoculture d'un mélange $\mathrm{H} 1, \mathrm{H} 2, \mathrm{H} 3$ et H4) appartiennent essentiellement au pathotype 710 qui ne peut se développer que sur l'hybride H1. II nous a semblé intéressant de comparer l'évolution des épidémies à l'intérieur de ces deux stratégies en fonction du taux de plantes appartenant à des génotypes sensibles. Le taux d'attaques primaires (telluriques) moyen observé sur l'hybride H1 est identique que celui-ci soit cultivé seul $(35,0 \%)$ ou en mélange $(34,8 \%)$. Par contre, le taux d'infections secondaires observé sur les individus $\mathrm{H} 1$ non infectés au niveau des racines varie, en moyenne sur les quatre années, de $36,2 \%$ lorsque $\mathrm{H} 1$ est cultivé seul à 13,8\% lorsqu'il est cultivé en mélange (figure 3 ).

\section{Discussion}

Pour analyser l'impact de la gestion des résistances monogéniques dominantes du tournesol au mildiou, nous avons développé un protocole de culture sous tunnel irrigué permettant d'obtenir sur un génotype sensible (hybride $\mathrm{H} 1$ sensible à la race 710 présente sur le site) des taux d'infection satisfaisants (> $35 \%$ ), et ce quelles que soient les conditions climatiques de l'année. Ce protocole confirme les observations réalisées en culture [28] sur l'importance, pour les infections racinaires, de la présence d'une grande quantité d'eau (présence d'eau libre dans les interstices du sol) durant la période de sensibilité maximale correspondant à la germination et à l'élongation de la radicule. Outre son intérêt pour notre étude, ce dispositif pourrait utilement être utilisé dans des programmes de sélection massale pour la résistance partielle au mildiou à l'image de ce qui a été développé pour Sclerotinia, Phomopsis et Botrytis [26, 29, 30].

L'observation des parcelles correspondant aux monocultures de génotypes ne possédant qu'un seul gène de résistance efficace (hybrides $\mathrm{H} 2$ et $\mathrm{H} 3$ résistants à la race 710 ) est tout à fait comparable aux observations réalisées en culture [14]. La perte de l'efficacité de la résistance par sélection de souches de Plasmopara halstedii virulentes, est, dans notre dispositif, accélérée par l'absence de rotation et par le maintien de conditions très favorables au parasite à chaque cycle de culture. Ces résultats confirment que l'utilisation d'une seule source de résistance majeure, si elle s'avère très efficace au début (moins de $3 \%$ d'attaques observées sur des génotypes non résistants à la race 710 qui sont sans doute des impuretés liées aux conditions de production de ces hybrides) s'effondre assez rapidement puisque dès la quatrième année on observe des taux d'attaque non négligeables (supérieurs à $15 \%$ ). De ce fait, notre couple Plasmopara halstedii/tournesol ne diffère pas des autres pathosystèmes pour lesquels les sélectionneurs ont exploité les gènes majeurs $[18,19]$. II a été démontré que les 


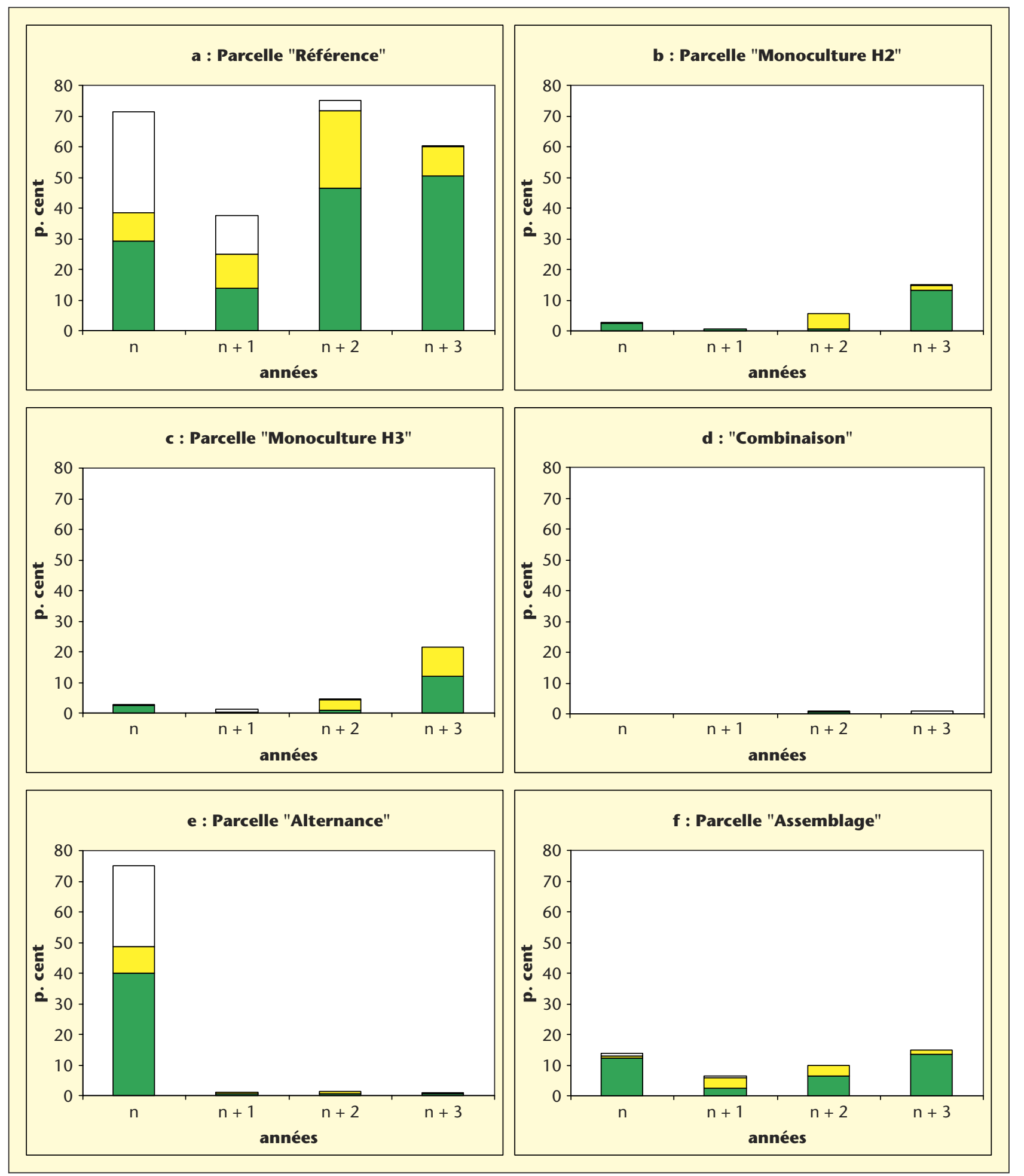

Figure 2. Evolutions des taux d'attaque de mildiou selon la gestion des gènes PI de résistance du tournesol : infections primaires (vert), infections secondaires systémiques (jaune), infections secondaires non systémiques (blanc).

gènes des deux sources de résistance (HA335 pour $\mathrm{H} 2$ et $\mathrm{RHA} 340$ pour $\mathrm{H} 3$ ) sont situés sur des chromosomes différents et sont liés à des RGA (resistance gene analogue) différents : TIR-NBSLRR pour HA335 et CC-NBS-LRR pour RHA340
$[16,22,23]$. Des travaux récents semblent également indiquer qu'ils conduisent à des réactions différentes, de type "hypersensibilité » pour la source « HA335 » [31] et « systémique acquise » pour la source «RHA340»
[32]. Ces différences expliquent, peut-être, celles qui sont observées dans notre expérimentation. Si l'efficacité de ces deux sources de résistance s'est effondrée après seulement 3 cycles de monoculture, des différences non négligea- 


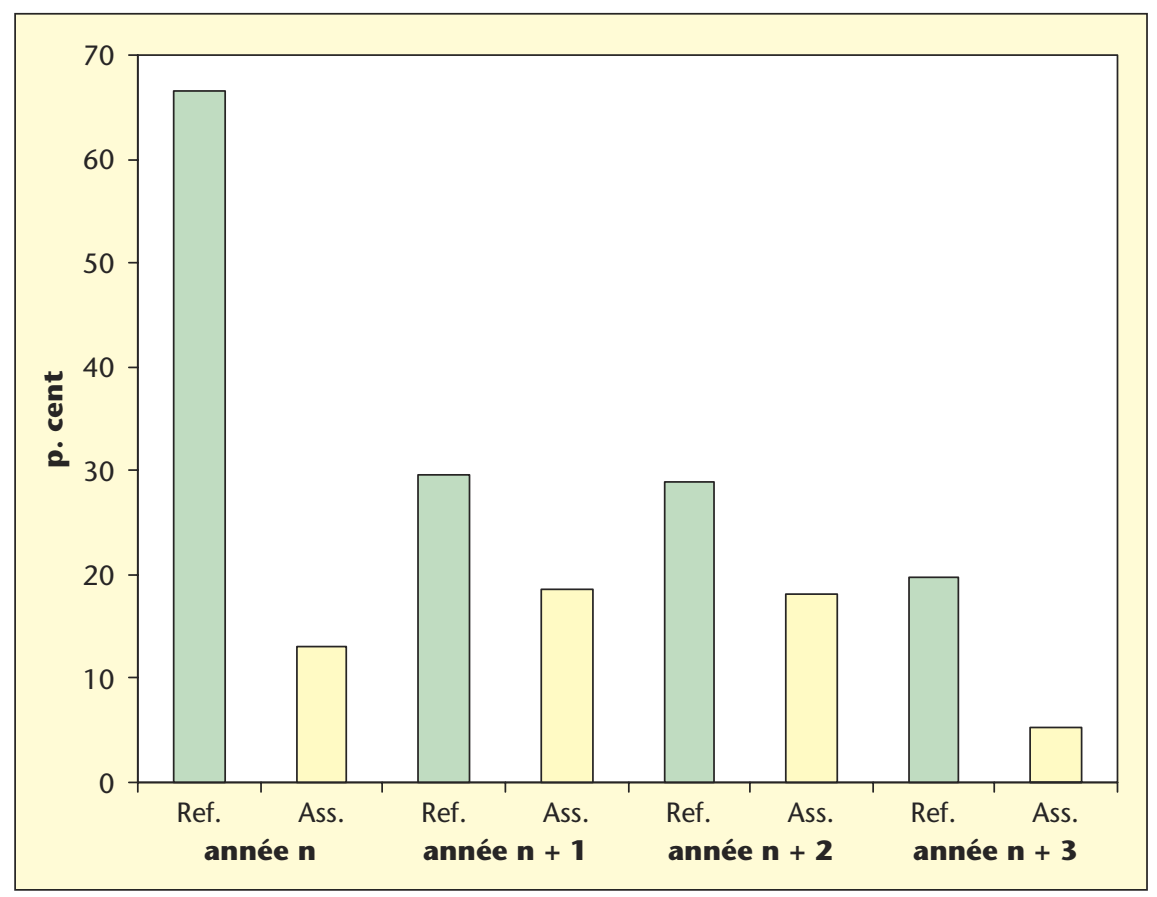

Figure 3. Comparaison des taux d'infections secondaires avec la race 710 de Plasmopara halstedii observés sur les pieds de tournesol de l'hybride H1 (sensible à la race 710) ayant échappé à l'infection primaire selon l'année d'expérimentation et le taux de plantes sensibles sur la parcelle.

Ref. = parcelle «Référence » avec 100 p.cent de H1. Ass. = parcelle « Assemblage » avec 25 p.cent de H1.

bles apparaissent l'année $n+3$ avec un taux de plantes malades plus élevé et une diversité de virulences plus importante avec $\mathrm{H} 3(21,4 \%$ et 4 pathotypes) qu'avec $\mathrm{H} 2(15,1 \%$ et seulement 2 pathotypes). La réaction d'hypersensibilité qui bloque très rapidement la progression du parasite dans les tissus de la plante s'avèrerait plus efficace (en termes de durabilité) que la réaction systémique acquise qui, chez le tournesol, permet au parasite de s'installer dans les tissus [32]. Ceci apparaît en contradiction avec l'hypothèse couramment admise qui affirme que plus la résistance est efficace et plus la pression de sélection sur le parasite est élevée. Ces observations mériteraient donc d'être confirmées dans un dispositif de laboratoire complètement contrôlé sur des lignées fixées pures.

II est intéressant à remarquer que les 4 nouvelles virulences découvertes dans notre expérimentation avaient déjà été isolées dans d'autres régions par les SPV : la race 304 en 2000, la race 314 en 2001 et les races 704 et 714 en 2002 (SPV, comm. pers.). Ce qui prouve bien que notre dispositif reproduit, à I'identique, ce qui se passe en culture. II n'est donc pas déraisonnable de penser que les races plus virulentes sont présentes à l'état résiduel dans la population indigène et que les gènes de résistance diminuent la fraction avirulente de la population parasite et sélectionnent parallèlement la fraction virulente.
Ce constat de l'effondrement de l'efficacité des résistances de type monogénique du tournesol, utilisées seules, vis-à-vis du mildiou qui a été observé en culture et confirmé dans notre expérimentation doit-il conduire la profession à I'abandon de ce type de résistance? Les analyses des autres stratégies de gestion de ces résistances monogéniques montrent qu'il est possible d'augmenter la durabilité de ces résistances:

- La combinaison de gènes («pyramidage ») qui consiste à associer dans un même génotype plusieurs gènes majeurs différents a été mise en œuvre dans l'hybride H4 qui cumule les deux sources de résistance (HA335 et RHA340) efficaces vis-à-vis de la race 710 . Contrairement aux monocultures de $\mathrm{H} 2$ et $\mathrm{H} 3$, aucune plante malade n'a été observée durant les deux premières années d'expérimentation, ceci est à relier au fait que, dans ce croisement, les deux parents possèdent un gène de résistance efficace, ainsi le risque de présence d'impureté ne possédant pas la résistance lors des productions de semences est-il fortement réduit. Ceci est confirmé par les tests de pureté réalisés avec les races 100 et 710 . Cette observation n'est pas sans importance car la présence dans une culture d'individus sensibles assure la multiplication de l'inoculum qui est confronté aux gènes de résistance, ce qui peut augmenter le risque d'apparition de souches présentant une nouvelle virulence. Malgré la présence de quel- ques pieds touchés en année $n+2$, le taux de plantes malades en année $n+3$ est extrêmement faible $(0,9 \%)$ par rapport aux taux observés lorsque les gènes sont utilisés seuls $(15,1$ et $21,4 \%)$. Dans notre expérimentation, la combinaison des deux sources de résistance qui présentent le même profil de résistance (tableau 4), est efficace soit parce que les souches de Plasmopara halstedii capables de surmonter ces deux gènes en même temps sont moins agressives, soit du fait de l'absence d'impureté sensible. La première hypothèse peut être vérifiée en analysant les isolats appartenants au pathotype 704 apparus sur cette parcelle à l'aide d'un protocole de mesure de l'agressivité et en les comparant aux isolats du même pathotype récoltés dans la monoculture H3. Pour confirmer la seconde hypothèse il serait envisageable de suivre l'évolution d'une même population parasitaire sur des parcelles suivies en monoculture de $\mathrm{H} 4$ d'une part et de $\mathrm{H} 4$ + quelques \% de $\mathrm{H} 1$ d'autre part.

La combinaison de gènes nécessite de disposer de nombreuses sources de résistance différentes. Actuellement seules deux sources différentes assurent la résistance à toutes les races présentes en France : P/8 situé sur le groupe de liaison 6 de la carte moléculaire Cartisol [17] et PIArg situé sur un autre groupe de liaison [24, 25]. II apparaît donc indispensable de rechercher dans les ressources génétiques (Helianthus annuus sauvages et Helianthus pérennes) de nouvelles résistances. De plus, si la combinaison de deux gènes est assez aisée pour le sélectionneur qui les apporte par chacun des deux parents, la gestion de trois gènes est beaucoup plus problématique car elle nécessite des marqueurs spécifiques à chaque gène (marqueurs moléculaires par exemple) afin de pouvoir suivre la combinaison de deux gènes ayant le même profil d'efficacité dans une lignée parentale. Enfin, les agriculteurs devraient disposer des informations leur permettant de choisir des variétés porteuses de ce type de combinaison si le risque « mildiou » est important, la simple information portant sur le profil d'efficacité de tel ou tel hybride vis-à-vis des races présentes en France ne suffit pas car elle ne distingue pas les génotypes qui possèdent une combinaison de gènes de résistance de ceux qui n'en possèdent qu'un.

- L'assemblage qui consiste à cultiver différentes génétiques en association a été mis en œuvre en mélangeant à parts égales nos quatre hybrides. La présence dans cet assemblage de $25 \%$ d'individus $\mathrm{H} 1$ sensibles à la race 710 explique les taux d'attaques relativement importants observés durant les 4 années d'expérimentation (de 6,5 à 15,1\%). Dans d'autres pathosystèmes, cette stratégie (multilignées, multihybrides, alternance dans les semis) est mise en œuvre afin de limiter les 
épidémies en réduisant l'inoculum source de nouvelles virulences et en bénéficiant d'un certain effet de protection (effet barrière) apporté par les individus résistants [33, 34]. Dans le cas du mildiou du tournesol, le processus infectieux primaire étant tellurique, le niveau d'infection d'un génotype sensible dépendra essentiellement du niveau d'infestation de la parcelle et des conditions climatiques favorables ou non. Les taux moyens d'attaque primaire du pathotype 710 observés sur l'hybride sensible $\mathrm{H} 1$ sont identiques que celui-ci soit cultivé seul ou en mélange. L'assemblage n'a donc aucun impact sur cette phase de l'épidémie. Par contre, si on s'intéresse aux infections aériennes, on remarque une réduction importante de celles-ci sur l'hybride $\mathrm{H} 1$ lorsque celui-ci est cultivé en mélange. Cette réduction peut être expliquée par la diminution de la quantité d'inoculum puisque le nombre de plantes malades sur lesquelles le parasite sporule est, en moyenne, quatre fois moins important que lorsque l'hybride $\mathrm{H} 1$ est cultivé seul. Cependant, on ne peut pas rejeter l'hypothèse de l'« effet barrière » même si notre dispositif de culture sous tunnel entraînait des conditions de confinement éloignées de celles rencontrées en culture. Un autre intérêt de I'assemblage incluant un génotype sensible est de limiter la pression de sélection sur le parasite permettant aux isolats non virulents sur les formes résistantes de se multiplier et de se maintenir sur la parcelle, intérêt contradictoire avec le fait que cela augmente la quantité d'inoculum confronté aux gènes de résistance. Avec l'apparition de la race 714 dans cette stratégie en 2004, les analyses prévues en 2005 doivent nous permettre de mesurer l'intérêt de maintenir une proportion d'individus sensibles. À l'exception de ces isolats 714 récoltés sur des infections tardives de la dernière année et qui pourraient être des contaminations issues d'individus étrangers à la parcelle, tous les isolats appartiennent au pathotype d'origine: race 710 . L'assemblage gagne donc en durabilité par rapport à la monoculture de gène. Dans le contexte actuel, I'assemblage nécessite cependant de disposer simultanément de plusieurs versions de la même variété et $d^{\prime}$ une identification claire du contenu du produit commercialisé. Ceci obligerait les obtenteurs à démultiplier les formes pour un même hybride, ce qui en plus du coût de la transformation des lignées parentales, poserait des problèmes difficilement surmontables dans la gestion de stocks de semences et dans la traçabilité des lots. D'autre part, la réglementation actuelle oblige un traitement anti-mildiou pour les semences non résistantes à toutes les races présentes sur le territoire français : se pose alors la question du statut de ces assemblages qui, par définition, incorporent des génétiques dif- férentes pour des profils de résistance différents. Ces assemblages devraient en effet intégrer, pour une faible proportion, un génotype dépourvu de gène efficace, ce qui permettrait de limiter encore l'extension de nouvelles races virulentes. Ceci nécessiterait d'étudier le taux de plantes «sensibles » acceptable d'un point de vue agronomique et sous-entend que l'agriculteur accepte un taux maîtrisé de plantes malades dans ses parcelles afin de réduire le risque d'apparition de nouveaux pathotypes qui pourraient fragiliser encore la culture du tournesol.

- L'alternance consiste à cultiver successivement différentes génétiques afin de diversifier la pression de sélection sur le parasite [35]. Mise en œuvre dans notre expérimentation, cette stratégie a permis de limiter les taux de plantes malades à moins de $1,5 \%$ les années $n+1, n+2$ et $n+3$, et cela malgré un niveau d'attaque très élevé l'année $n(75,1 \%)$ donc un niveau d'infestation de la parcelle également très élevé. L'intérêt d'une telle stratégie réside dans le fait que les isolats sélectionnés une année sont confrontés à une nouvelle source de résistance au cycle suivant. Ainsi, tous les isolats récoltés les trois premières années appartiennent au pathotype 710 alors que des isolats appartenant au pathotypes 704 et 714 étaient isolés dans les parcelles de monoculture des hybrides $\mathrm{H} 2$ et $\mathrm{H} 3$. L'isolement de souches appartenant au pathotype 714 sur des plantes infectées tardivement (infections aériennes) l'année $n+4$ place I'hybride $\mathrm{H} 4$ dans une situation en tout point identique à celle qui est observée la même année dans la parcelle de monoculture de combinaison de gène. Actuellement, il n'est pas possible de conclure sur une éventuelle perte $d^{\prime}$ efficacité ou sur une pollution intempestive. Pour le mildiou du tournesol dont les infections primaires (les plus dommageables) sont telluriques, cette alternance devrait être raisonnée à la parcelle : ne pas cultiver sur la même parcelle des génotypes qui ont les mêmes gènes de résistance.

L'utilisation de cette stratégie nécessite la transparence sur les génétiques utilisées, afin que I'agriculteur puisse raisonner son choix variétal en fonction des gènes présents dans les variétés commerciales. La simple information sur le profil de résistance global des variétés n'est pas suffisante pour ce raisonnement et ne lui permettra pas d'alterner les sources de résistance sur son exploitation. L'investissement (coût de sélection, de production et de gestion des semences) des obtenteurs pour l'inscription au catalogue de différentes versions du même hybride pourrait être valorisé, puisque le choix des sources de résistance par les producteurs pourrait se faire sans changer de variété. L'absence de ces différentes formes d'un même hybride commercial serait un frein puissant à la mise en place de l'alternance car celle-ci obligerait les agriculteurs à « zapper » entre obtenteurs, démarche qui ne pourra pas se faire avec l'appui de ces derniers. L'alternance pourrait être envisagée à une autre échelle, celle d'un bassin de production par exemple. Cette stratégie régionale pourrait, comme cela a été fait dans le cas de la lutte contre les rouilles des céréales au Royaume-Uni, être basée sur une recommandation du CETIOM qui prendrait en compte les informations sur le risque «mildiou » issues des prospections des SPV dans le cadre de la lutte contre Plasmopara halstedii, parasite de quarantaine et celles fournies par les obtenteurs et/ou la recherche sur les sources de résistance présentes dans les variétés commerciales. Enfin, des études supplémentaires sont nécessaires pour définir s'il est possible, sans risque insupportable pour l'agriculteur, de revenir à une source de résistance contournée après plusieurs alternances.

Une autre stratégie ayant pour objectif l'augmentation de la durabilité des gènes $P /$ consisterait à compléter ces résistances par une résistance générale. Castro et al. [36] ont démontré qu'il était possible d'associer résistance majeure et résistance partielle dans le cas de la rouille de l'orge. L'une des principales difficultés rencontrées dans ce type de combinaison est le fait que la présence d'un ou de plusieurs gènes majeurs masque la résistance partielle. Les marqueurs moléculaires prennent donc ici tous leurs intérêts. Pour le couple Plasmopara halstedii/tournesol, les expérimentations réalisées ces deux dernières années ont démontré qu'une résistance partielle non race spécifique existe dans le tournesol cultivé et qu'elle est exploitable en sélection [37]. Cette résistance est efficace sur toutes les races, cependant elle n'apparaît pas suffisante en agriculture si les conditions pédoclimatiques sont très favorables au parasite. Son utilisation en sélection en complément des gènes $P /$ permettrait de limiter l'extension rapide des nouvelles races en prolongeant l'intérêt agronomique de telle ou telle variété.

Enfin, il n'est pas raisonnable de parler de résistance durable si nous n'intégrons pas à la gestion des résistances, qu'elles soient complètes race spécifiques ou partielles, les autres stratégies de lutte. En premier lieu, les conséquences de l'introduction de pathotypes américains à la fin des années 80 montrent, si besoin en était, l'importance de contrôler d'éventuelles nouvelles introductions. Le statut de "parasite de quarantaine » de Plasmopara halstedii nous permet de bénéficier d'une réglementation à I'importation stricte qu'il nous faut respecter afin de limiter au maximum l'introduction de souches à partir d'autres continents qui pourraient présenter une nouvelle virulence ou 
apporter de la variabilité dans les populations indigènes par brassage génétique. De même, les agriculteurs doivent, par leurs techniques culturales, limiter au maximum les risques " mildiou », en particulier en évitant les semis directs qui favorisent la maladie [38] et en respectant des délais dans le retour d'un tournesol sur une parcelle fortement contaminée par le parasite. En effet, il a été démontré un lien étroit qui existe entre le niveau d'infestation d'une parcelle et le nombre de plantes malades présentes sur cette parcelle les années $n-1$ et $n-2$ [13]. De plus, si Plasmopara halstedii peut lever sa sensibilité au méfénoxam (isomère du métalaxyl) qui est le seul antimildiou systémique actuellement autorisé en traitement de semence sur tournesol [39], il a également été démontré [14] que cette résistance au produit était réversible, ce qui doit nous conduire à n'utiliser cette molécule qu'à bon escient. Ce produit ne devrait être employé que lorsque le risque «mildiou » est élevé, ce qui dépend de l'historique de la parcelle, de la présence ou non de nouvelles virulences dans le bassin de production et bien sûr du choix variétal. Malheureusement, cette gestion raisonnable de la lutte chimique s'oppose à un décret instaurant, en France, le traitement obligatoire de toutes les semences des variétés non déclarées officiellement résistantes à toutes les races reconnues présentes sur le territoire français. De ce fait et en absence de liste officielle pour les 5 nouvelles races déclarées présentes en 2004, toutes les variétés de tournesol commercialisées en France en 2005, sont traitées avec du méfénoxam quels que soient leur profil de résistance et leur lieu d'implantation. Une réelle protection intégrée, à l'image de ce qui est proposé pour d'autres pathosystèmes [34, 40-42], contre le mildiou du tournesol faisant appel à des méthodes de préservation des résistances, des pratiques culturales adaptées et des moyens chimiques raisonnés, devrait permettre d'augmenter la durée d'efficacité des résistances variétales.

Remerciements. Les auteurs tiennent à remercier les stagiaires (C. Albert, A. Belkorchia et $P$. Pruvost) ayant participé à cette expérimentation. Nous remercions l'AMSOL pour la fourniture gracieuse des semences des hybrides et pour sa participation à l'élaboration de ce travail et M.C. Tardin et $F$. Vear pour une lecture critique de cet article. Ce programme a bénéficié d'un soutien du ministère français de l'Agriculture: Convention MCB-13A-Conv01-61.21.43 CETIOM-Tournesol/ Mildiou.

\section{RÉFÉRENCES}

1. LEPPIK EE. Distribution of downy mildew and some others seed-borne pathogens on sunflower. Bull FAO 1962; 10 : 126-9.
2. TOURVIEILLE de LABROUHE D. La nouvelle nomenclature des races de Plasmopara halstedii, agent du mildiou du tournesol, appliquée aux races françaises. OCL $1999 ; 6: 219-22$.

3. LECLERCQ P, CAUDERON Y, DAUGE M. Sélection pour la résistance au mildiou du tournesol à partir d'hybrides topinambour $\times$ tournesol. Ann Amélior Plantes 1970 ; 20 : 363-73.

4. VEAR F. L'évolution des variétés de tournesol. Circuit cult 1982 : $94: 60-1$.

5. TOURVIEILLE de LABROUHE D, MOUZEYAR $S$, LAFON $S$, et al. Evolution des races de mildiou (Plasmopara helianthi) sur tournesol, en France. $3^{e}$ Conf. Int. sur les Maladies des Plantes. 3-5 décembre 1991, Bordeaux (France) : 777-84.

6. TOURVIEILLE J, WALSER P, TOURVIEILLE de LABROUHE D. Distribution et caractérisation des races de mildiou du tournesol en France, bilan 1993. Rencontres annuelles du CETIOM. 30 novembre-3 décembre 1993, Paris (France) : 29-35.

7. LAFON S, PENAUD A, WALSER P, et al. Le mildiou du tournesol toujours sous surveillance. Phytoma-La défense des cultures 1996 : 35-6.

8. PENAUD A, LAFON S, TOURVIEILLE J, et al. Evolution du mildiou du tournesol en France. $5^{e}$ Conf. Int. sur les Maladies des Plantes, 3-5 décembre 1997, Tours (France) : 407-12.

9. LAFON S, DELOS M, RAULICI, et al. Bilan de dix années de surveillance du mildiou du tournesol en France. $15^{e}$ Conf Int sur le Tournesol. 12-15 juin 2000, Toulouse (France) : 149-54.

10. PENAUD A, MOINARD ], MOLINERO-DEMILLY $V$, et al. Evolution du Mildiou du tournesol en France : le point sur les dernières données du réseau de surveillance. AFPP $-7^{e}$ Conf Int sur les Maladies des Plantes. 3-5 décembre 2003, Tours (France), CDrom.

11. GULYA TL, URS NVRR. A new race of sunflower downy mildew. Phytopathol $1985 ; 75: 1339$.

12. ROECKEL-DREVET P, TOURVIEILLE J, GULYA T], et al. Molecular variability of sunflower downy mildew, Plasmopara halstedii, from different continents. Can / Microbiol 2003 ; 49: 492502.

13. TOURVIEILLE de LABROUHE D.Faire durer la résistance au mildiou du tournesol. Rencontres Annuelles du CETIOM. 30 novembre-1 décembre 2004, Paris (France) : 67-73.

14. TOURVIEILLE de LABROUHE D. El Mildiu de Girasol, manejar el control en pos de una eficacia durable. $S^{\circ}$ Congreso Argentino de Girasol, 12 y 13 de Agosto de 2003, BA (Argentina): 63-70, 2004

15. MOUZEYAR S, ROECKEL-DREVET P, GENTZBITTEL L, et al. RFLP and RAPD mapping of sunflower P/1 locus for resistance to Plasmopara halstedii race 1. Theor Appl Genet $1995 ; 91$ : 733-7.
16. ROECKEL-DREVET P, GAGNE G, MOUZEYAR S, et al. Colocation of downy mildew (Plasmopara halstedii) resistance genes in sunflower (Helianthus annuus). Euphytica 1996 ; 91 : 225-8.

17. BERT PF, TOURVIEILLE de LABROUHED, PHILIPPON ], et al. Identification of a second linkage group carrying genes controlling resistance to downy mildew (Plasmopara halstedii) in sunflower (Helianthus annuus L.). Theor Appl Genet $2001 ; 103$ : 992-7.

18. DE VALLAVIEILLE-POPE C. Management of disease resistance diversity of cultivars of a species in single fields : controlling epidemics. $C R$ Biologies $2004 ; 327: 611-20$

19. MCDONALD BA, LINDEC. The population genetics of plant pathogens and breeding strategies for durable resistance. Euphytica 2002 ; $124: 163-80$.

20. ZHU Y, CHEN H, FAN J, et al. Genetic diversity and disease control in rice. Nature 2000 ; 406 : 718-22.

21. VEAR F, PHILIPPON I, ROCHE $S$, et al. Genetical analyses of the sunflower downy mildew resistance gene PI5. $15^{e}$ Conf Int sur le Tournesol, Toulouse (France), II, 12-15 juin 2000 : J31-J36.

22. BOUZIDI MF, BADAOUI S, CAMBON F, et al. Molecular analysis of a major locus for resistance to downy mildew in sunflower with specific PCR-based markers. Theor Appl Genet $2002 ; 104: 592-600$

23. RADWAN O, BOUZIDI MF, VEAR F, et al. Identification of non-TIR-NBS-LRR markers linked to the $P / 5 / P / 8$ locus for resistance to downy mildew in sunflower. Theor Appl Genet 2002; 106 : 1438-46.

24. VEAR F, TOURVIEILLE de LABROUHE D, MILLER JF. Inheritance of the wide-range downy mildew resistance in the sunflower line Rha419. Helia $2003 ; 26: 19-24$.

25. DUSSLE CM, HAHN V, KNAPP V, BAUER E. PIArg from Helianthus argophyllus is unlinked to other downy mildew resistance genes in sunflower. Theor Appl Genet 2004 ; 109 : 1083-6.

26. TOURVIEILLE de LABROUHE D, VEAR F, HABOUZIT J. Culture du tournesol sous tunnel en filet avec humectation contrôlée pour l'étude du Sclerotinia sclerotiorum. Inf Tech CETIOM $1986 ; 96$ : 20-8.

27. TOURVIEILLE de LABROUHE D, DUCHER $M$, PHILIPPON J, et al. Les méthodes d'analyse du mildiou. In : D. Tourvieille de Labrouhe et al. (eds), Le mildiou du tournesol. INRA-Editions: 53-66, 2000.

28. DELOS M, EYCHENNE N, BIRBA I, FABRY C. Etude des facteurs expliquant les fluctuations des attaques de Plasmopara halstedii en France. $6^{e}$ Conf Int sur les Maladies des Plantes, 6-8 décembre 2000, Tours (France), vol. $1: 259$ 66. 
29. TOURVIEILle de LABROUHe D, PELletier C. Jugement de la résistance du tournesol au phomopsis sous tunnel en filet avec humectation contrôlée. Inf Tech CETIOM 1988 ; 103 : 28-9.

30. LADSOUS O, VEAR F, TOURVIEILLE de LABROUHE D. Heredity of resistance to Botrytis cinerea in sunflower (Helianthus annuus) measured by artificial infections. Plant Breeding $1991 ; 107: 235-41$.

31. MOUZEYAR S. Unraveling the molecular mechanisms of pathogen resistance in sunflowers. $15^{e}$ Conf Int sur le Tournesol, Toulouse (France), I, 12-15 juin 2000, PI.D8-PI.D15.

32. RADWAN O, MOUZEYAR S, NICOLAS $P$, et al. Induction of a sunflower CC-NBS-LRR resistance gene analogue during incompatible interaction with Plasmopara halstedii. J Exp Bot 2005 ; (in press).

33. LANNOU C, MUNDT CC. Evolution of a pathogen population in host mixtures : rate of emergence of complex races. Theor Appl Genet 1997 ; 94 : 991-9.
34. MUNDT CC, COWGER C, GARRETT K. Relevance of integrated management to disease durability. Euphytica $2002 ; 124$ : 245-52.

35. HOLT I, CHANCELOR TCB. Modelling the spatio-temporal deployment of resistant varieties to reduce the incidence of rice tungro disease in a dynamic cropping system. Plant Pathol 1999 ; 48 : 453-61.

36. CASTRO AJ, CAPETTINIF, COREY AE, et al. Mapping and pyramiding of qualitative and quantitative resistance to stripe rust in barley. Theor Appl Genet 2003 ; 107 : 922-30.

37. TOURVIEILLE de LABROUHE D, TARDIN MC, MESTRIES E, et al. Partial, non-race specific resistance to downy mildew in cultivated sunflower lines. 16th Int Sunflower Conf, August 29-September 2, 2004, Fargo (ND, USA) : 10510.

38. CALVIÑO P, PEREYRA V, ESCANDE A. Influencia de la siembra directa sobre la cantidad de plantas con sintomatología de mildiu, (Plasmopara halstedii (Farl)). E-campo.com - el futuro esta en la tierra 2005, 27-ene-05.
39. ALBOURIE JM, TOURVIEILLE J, TOURVIEIILE DE LABROUHE D. Resistance to metalaxyl in isolates of the sunflower pathogen Plasmopara halstedii. Eur J Plant Pathol 1998 ; 104 : 235-42.

40. MCDONALD BA, LINDE C. Pathogen population genetics, evolutionary potential, and durable resistance. Annu Rev Phytopathol $2003 ; 40$ : 349-79.

41. PARLEVLIET JE. Durability of resistance against fungal, bacterial and viral pathogens : present situation. Euphytica $2002 ; 124: 147-56$.

42. PINK DAC. Strategies using genes for nondurable disease resistance. Euphytica 2002; $124: 227-36$. 